\title{
Transition bei chronischen Erkrankungen der Kindheit und Jugend
}

\section{Einleitung}

\section{Epidemiologie}

Chronische Erkrankungen, Behinderungen und Gesundheitsrisiken (engl. „chronic conditions") stellen in der Adoleszenz eine grosse Herausforderung für den Jugendlichen, seine Familie und die betreuenden Fachpersonen dar. Deren Häufigkeit ist aufgrund unterschiedlicher Methoden und Definitionen schwierig zu bezeichnen. Befragungen von Jugendlichen lassen schätzen, dass rund $7 \%$ bis $15 \%$ betroffen sind. Darunter fallen sicherlich Jugendliche mit Einschränkungen im Alltag, beispielsweise durch Diabetes und Epilepsie, bei grosszügigen Einschlusskriterien aber auch Jugendliche mit mildem Asthma und Fehlsichtigkeiten des Auges. Die Wahrscheinlichkeit einer Erkrankung scheint insgesamt höher zu sein bei männlichen Jugendlichen, tiefem sozialem Status und tiefer Bildung. Sie variiert je nach Entität auch stark zwischen Ländern und Weltregionen. Dank Fortschritten in der Behandlung und Betreuung leben heute junge Erwachsene mit einer „chronic condition“ länger als je zuvor: 90\% erreichen die dritte Lebensdekade.[1]

Die Herausforderungen der "chronic conditions“ (nachfolgend sinngemäss als "chronische Erkrankung" zusammengefasst) entstehen aus einer gegenseitigen Wechselwirkung von Entwicklung und Erkrankung: Die Entwicklung in der Adoleszenz ist eine Zeit grosser physiologischer Änderungen und sozialer Prozesse, die sowohl durch die Erkrankung beeinflusst wird, als auch Auswirkungen auf die Erkrankung hat. Durch die Erkrankung beeinflusst sind biologische Entwicklungsaspekte beispielsweise im Sinne einer verzögerten Pubertät oder reduzierten Knochendichte, psychologische im Sinne einer Ablösungsstörung oder Übernahme einer Krankheitsrolle, und soziale im Sinne einer Isolation oder Einschränkung der Berufswahl. Typische Auswirkungen der Entwicklung auf die Erkrankung sind hormonell beeinflusste metabolische Prozesse, die infrage gestellte Compliance der Medikamenteneinnahme, und Risikoverhalten.

Diese Herausforderungen sind einerseits spezifisch für bestimmte Gruppen von betroffenen Jugendlichen, sie sind aber auch übergreifend die gleichen. Sie führen zur Aufgabe an medizinische Fachpersonen eine gute Langzeitbetreuung dieser Menschen anzubieten: Wer benötigt eine Betreuung? Wie ist sie organisiert? Warum ist sie notwendig?

\section{Wozu Transition - Beispiel Krebs}

In Westeuropa erkrankt ungefähr eines von 500 Kindern innerhalb der ersten 15 Lebensjahre an Krebs. Dies entspricht in der Schweiz 200 Kinderkrebsdiagnosen pro 
Published in final edited form as:

Pädiatrie up2date 2016; 11(01): 55-74 DOI: 10.1055/s-0041-107502

Jahr. Am häufigsten tritt in die Erkrankung in den ersten 5 Jahren auf. Jungen erkranken ungefähr 1,2-mal häufiger als Mädchen. Die häufigste diagnostische Gruppe ist die akute Leukämie (rund 34\% aller Erkrankungen), gefolgt von Tumoren des zentralen Nervensystems (19\%) und Lymphomen (13\%). Therapeutisch eingesetzt werden neben chirurgischen Eingriffen Chemotherapie, Radiotherapie und Stammzelltransplantationen. Die Zusammensetzung und Dosierung dieser Therapieoptionen wurde dank weltumspannenden Forschungsnetzen derart verbessert, dass heute rund $83 \%$ der Patienten mindestens fünf Jahre nach Diagnose am Leben sind, wobei zwischen den Diagnosen grosse Unterschiede bestehen (akute lymphoblastische Leukämie: 90\%, Weichteilsarkome: 70\%).[2]

Berechtigterweise kann sich die Kinderonkologie über diese Erfolge freuen. Allerdings treten bei drei von fünf Langzeitüberlebenden Spätfolgen auf, Nebenwirkungen, welche fünf Jahre nach Therapieabschluss bestehen bleiben oder neu entstehen. Spätfolgen umfassen biologische, psychische und soziale Probleme (Tabelle 1). Ursachen sind hochdosierte Chemo- und Radiotherapien, welche Kollateralschäden in gesundem Gewebe hinterlassen, das Wachstum eines Tumors in gesundes Gewebe, und Krebs als „life-event“, der das System einer Familie erschüttert. Die Auswirkungen der Spätfolgen reichen von „lebenseinschränkend“ bis lebensgefährlich.

Merke:

Spätfolgen der Therapie gegen Krebs im Kindesalter treten oft erst nach Jahrzehnten auf. Forscher vermuten heute, dass das relative Risiko im Vergleich zu gleichaltrigen Erwachsenen kein Plateau erreicht, sondern über Jahrzehnte zunimmt.

\begin{tabular}{|l|l|}
\hline $\begin{array}{l}\text { Tabelle 1 } \\
\text { Häufige Spätfolgen nach Krebs im Kindesalter[3] }\end{array}$ \\
\hline Spätfolge & Wichtigster Risikofaktor \\
\hline Zweittumoren & Bestrahlung, insbesondere kranial \\
\hline Kardiomyopathien & Hoch-dosierte Anthrazykline \\
\hline $\begin{array}{l}\text { Übergewicht und } \\
\text { metabolisches Syndrom }\end{array}$ & Kraniale Bestrahlung, Glukokortikoide \\
\hline $\begin{array}{l}\text { Zerebrovaskuläre } \\
\text { Erkrankungen }\end{array}$ & Kraniale Bestrahlung, intrathekale Therapien \\
\hline Osteopenie/Osteoporose & Kraniale Bestrahlung, Methothrexat, Glukokortikoide \\
\hline $\begin{array}{l}\text { Wachstumshormonmangel } \\
\text { und neurokognitive } \\
\text { Defizite }\end{array}$ & Kraniale Bestrahlung \\
\hline Katarakte & \\
\hline $\begin{array}{l}\text { Psychosoziale } \\
\text { Schwierigkeiten }\end{array}$ & $\begin{array}{l}\text { Äusserlich sichtbare Veränderungen durch } \\
\text { Operationen bei Hirntumoren, Amputationen bei } \\
\text { Knochentumoren }\end{array}$ \\
\hline
\end{tabular}


Published in final edited form as:

Pädiatrie up2date 2016; 11(01): 55-74 DOI: 10.1055/s-0041-107502

Kinder, Jugendliche und jungen Erwachsene nach einer Krebserkrankung, nennen sich „Survivors“. Survivors sind nicht mehr Patienten und leiden meist auch nicht unter einer Behinderung; aber sie leiden unter Spätfolgen und einem Risiko für Spätfolgen, entstanden aus der Erkrankung und Behandlung in ihrer Kindheit. Sie sollten nachbetreut werden, um Spätfolgen verhindern oder frühzeitig entdecken und behandeln zu können. Im deutschsprachigen Raum wurde dieses Thema in den letzten Jahren zunehmenden erforscht und einige spezialisierte Nachsorgezentren an Zentrumsspitälern ergänzen mittlerweile „biologische“ Kontrollen (Suche eines Krebsrückfalls in den ersten Jahren nach der Erkrankung). Sie bieten langfristige, auf die Bedürfnisse der Survivors zugeschnittene Programme an. Aus Register- und Befragungsdaten in der Schweiz ist bekannt, dass bisher über die Hälfte aller Survivors im Jugendalter aus der Nachsorge ausschied und nur ein geringer Anteil an einem derartigen Programm teilnehmen konnte.

Ein wichtiger Grundsatz der Nachsorge ist die Stratifizierung aufgrund des Risikos für Spätfolgen. Bei geringer Exposition kann ein Survivor von einem Grundversorger nachbetreut werden, der über die Erkrankung, Behandlung und Nachsorge informiert ist. Öffentlich zugängliche Richtlinien der Fachspezialisten vereinfachen die Entscheidung, ob und in welcher Frequenz beispielsweise das Herz bei AnthrazyklinExposition mittels Ultraschall untersucht werden sollte.[4, 5]

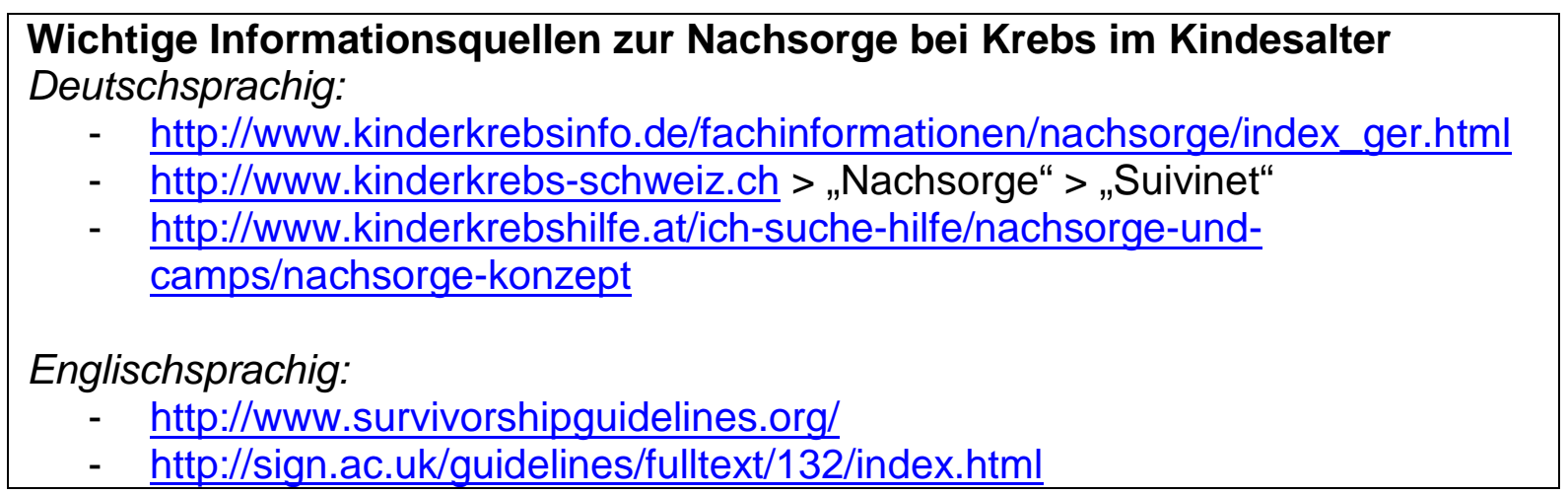

\section{Kasuistik 1}

Eine 16-jährige junge Frau besucht eine jugendmedizinische Praxis. Ihre Geschichte beinhaltet die Diagnose einer akuten lymphatischen Leukämie im Alter von drei Jahren. Sie weiss nicht, welche Therapien sie erhalten hat, aber ihre Eltern haben ihr mitgeteilt, dass sie während zwei Jahren behandelt wurde und sie nie einen Rückfall erlitten hat. Sie hat die Sekundarschule abgeschlossen und ist in Ausbildung zu einer Bankfachangestellten. Sie ist seit vielen Jahren nicht mehr in Betreuung bei der damals behandelnden Kinderklinik. Aktuell ist die Frau frei von Beschwerden bei seit früher Kindheit bestehender Adipositas.

Der Pädiater fordert bei der Kinderklinik eine Zusammenfassung der Behandlung und vorgeschlagenen Nachsorge an. Er bedenkt, dass bei einer kranialen Bestrahlung erhöhte Risiken für Zweittumoren, zerebrovaskuläre Ereignisse, 
Published in final edited form as:

Pädiatrie up2date 2016; 11(01): 55-74 DOI: 10.1055/s-0041-107502

Wachstumshormonmangel und neurokognitive Defizite bestehen. Er überprüft BMI, Blutdruck und Lipide aufgrund der Exposition mit Glukokortikoiden. Er zieht eine Knochendichtemessung in Erwägung und überweist die junge Frau einem Kardiologen zur Ultraschalluntersuchung des Herzen wegen des Risikos einer linksventrikulären Dysfunktion.

\section{Wozu Transition - Beispiel angeborene Herzfehler}

In westlichen Ländern liegt die Häufigkeit angeborener Herzfehler (AHF) seit mehr als 30 Jahren stabil bei ca. 0.5-1\% aller Lebendgeburten. Starben Mitte des letzten Jahrhunderts noch 60-70\% aller Betroffenen im Kindesalter, so erreichen mittlerweile neun von zehn das Erwachsenenalter. Zudem werden Erwachsene mit AHF je länger je älter. Diese Entwicklungen haben dazu geführt, dass seit der Jahrtausendwende mehr Erwachsene als Kinder mit AHF in den hoch industrialisierten Ländern leben.[6]

Die medizinischen Errungenschaften, insbesondere die Fortschritte der HerzChirurgie, haben im ersten Moment die Hoffnung aufkommen lassen, dass Kinder mit Herzfehler durch einen Eingriff „geheilt“ werden könnten. Begriffe wie „Totalkorrektur“ wurden geprägt, ohne wissen zu können, dass der Langzeitverlauf ungünstiger sein wird, als eine „totale Korrektur" auf den ersten Blick erwarten liesse. Die Erfahrungen der letzten 30 Jahre haben gezeigt, dass die wenigsten Herzfehler durch Eingriffe "geheilt" oder „total korrigiert" werden können. Die Gründe dazu sind vielfältig und bestehen aus den Folgen (Sequelae) und residuellen (Residuae) Befunden des Herzfehlers selbst und der zur Korrektur nötigen Interventionen. Die häufigsten Gründe für kardiovaskuläre Komplikationen nach Herzfehler-Eingriffen sind:

- $\quad$ kardiales Narbengewebe respektive chirurgische Nähte auf atrialer oder ventrikulärer Ebene dienen als arryhthmogenes Substrat

- biologische Klappen, Conduits oder andere Implantate degenerieren und benötigen Re-Operationen

- je nach Läsion und Operation besteht ein erhöhtes Endokarditis-Risiko

- funktionell korrigierende Eingriffe bei komplexen Herzfehlern (z.B. die FontanZirkulation beim univentrikulären Herzen oder die Vorhofsumkehr bei der kompletten Transposition der grossen Gefässe) führen im Langzeitverlauf zu einer Herzinsuffizienz

- neurologische oder andere nicht-kardiale Defizite nach Eingriffen oder KoMorbiditäten als Teil eines begleitenden Syndrom-Komplexes können die Lebenserwartung weiter beeinträchtigen.

Diese Liste ist nicht abschliessend. Tatsache ist, dass selbst bei einfachen Herzfehlern, wie z.B. ein Ventrikelseptumdefekt, die Lebenserwartung 30-40 Jahren nach der Operation geringer ist als in der Normalbevölkerung und die Morbidität deutlich erhöht ist.[7] 
Published in final edited form as:

Pädiatrie up2date 2016; 11(01): 55-74 DOI: 10.1055/s-0041-107502

Merke:

Langzeit-Komplikationen treten nicht nur bei korrigierten komplexen Herzfehlern auf, sondern sind auch bei „einfachen“ Herzfehlern zu beobachten sind, wie z.B. einem Ventrikelseptumdefekt (VSD)

Dies erklärt, weswegen auch Erwachsene mit AHF, trotz erfolgreicher Behandlung in der Vergangenheit, eine kontinuierliche, spezialisierte medizinische Betreuung benötigen. Diese Erkenntnis ist hauptsächlich in den letzten 10-20 Jahren gereift und hat zur Folge, dass ein Grossteil der heutigen älteren Erwachsenen mit AHF der Kindermedizin „entwachsen“ sind mit der Vorstellung geheilt zu sein. Zudem war vor 30 Jahren keine ausreichend qualifizierte Infrastruktur auf Erwachsenenseite vorhanden, die eine Nachkontrolle hätte sicherstellen können. Aus diesen Gründen sind etliche ältere Erwachsene mit AHF heutzutage unzureichend betreut, ohne sich dessen bewusst zu sein.

Seit der Jahrtausendwende hat sich in Deutschland, Österreich und der Schweiz die Zahl spezialisierter Zentren für die Betreuung von Erwachsenen mit AHF vervielfacht. Allein in der Schweiz ist sie zwischen 1995 und 2015 von einem universitären auf 5 solcher Zentren angewachsen, ergänzt durch weitere Sprechstunden in grösseren nicht-akademischen Kliniken. Dennoch lässt die Anzahl betreuter Patienten vermuten, dass die Mehrheit aller Betroffenen nicht auf diese Angebote zurückgreift.[8] Die Gründe dazu sind vielfältig. Nebst dem fehlendem Bewusstsein gibt es auch jüngere Patienten die sich einer kardiologischen Verlaufskontrolle im Erwachsenenalter bewusst entziehen, aus Angst vor schlechten Nachrichten oder um die Tatsache des Herzfehlers soweit als möglich zu verdrängen. Die Prädiktoren, welche Erwachsene ohne adäquate Nachbetreuung auszeichnen, werden in Kasuistik 2 exemplarisch dargestellt. Es sind dies:

- Letzter Eingriff in der frühen Kindheit

- Höheres Alter respektive Kindheit in einer früheren Dekade

- Keine Empfehlungen bezüglich Nachkontrollen anlässlich des letzten kinderkardiologischen Kontrolle

- Frühe Selbständigkeit, früher Auszug aus dem Elternhaus

- Tiefes Einkommen

- Männliches Geschlecht

- Geringes medizinisches Verständnis hinsichtlich des Herzfehlers

Diese fehlende Nachbetreuung hat Konsequenzen. Erwachsene mit AHF suchen doppelt so häufig den Notfall auf verglichen mit der Normalbevölkerung, und insbesondere ab dem 40. Lebensjahr steigt diese Zahl überproportional an. Dies widerspiegelt die mit dem Alter zunehmende Häufigkeit kardialer Komplikationen. Einer von 4 Erwachsenen wird in einem 5-Jahres-Intervall mindestens einmal hospitalisiert und in 2 von 5 Fällen in einer Notfallsituation.[8] Erwachsene mit AHF ohne kontinuierliche Nachbetreuung sind die am meisten gefährdeten Patienten für 
Published in final edited form as:

Pädiatrie up2date 2016; 11(01): 55-74 DOI: 10.1055/s-0041-107502

kardiale Notfälle. Sie haben ein 10-fach erhöhtes Risiko relevante kardiale Probleme zu entwickeln und ein 3-fach erhöhtes Risiko notfallmässig einen Eingriff zu benötigen, verglichen mit Erwachsenen mit AHF und kontinuierlicher Betreuung.

\section{Kasuistik 2}

Ein 27-jähriger Mann, der eine Lehre zum Servicefachangestellten absolviert hat und nun im Pflegebereich eine Ausbildung begonnen hat, meldet sich beim Wohnspital aufgrund zunehmender Müdigkeit. Er wurde in der Kindheit aufgrund eines komplexen Herzfehlers (siehe Graphik: Double outlet right ventricle mit subpulmonalem VSD, Malposition der grossen Gefässe, Straddling der Trikuspidalklappe) operiert. Nach einem ersten palliativen Eingriff im dritten Lebensmonat wurde im Alter von 7 Jahren eine Fontan-Zirkulation angelegt. Er wird vom Spital-Kardiologen in eine spezialisierte Sprechstunde überwiesen. Aus der Zeit des Eingriffes ist ihm noch in Erinnerung, wie er sich am Morgen vor dem geplanten Spitaleintritt zur Fontan-Operation im Elternhaus unter dem Bett versteckt hat, um dem Spitalaufenthalt zu entgehen. Postoperativ kommt es zu einer Endokarditis, die medikamentös behandelt werden kann. Eine retrosternale Hautfistel wird ein Jahr später chirurgisch revidiert. Aufgrund der perioperativ verabreichten Blutprodukte infiziert sich der Mann mit einer Hepatitis C.

Klinisch verbessert sich seine Leistungsfähigkeit und die letzte kinderkardiologische Verlaufskontrolle findet 5 Jahre postoperativ, im 12. Lebensjahr, statt. Seitdem wurden keine Kontrollen mehr durchgeführt, einerseits weil kein Aufgebot erfolgte, andererseits weil aus Patientensicht kein weitere Handlungsbedarf bestand.

Die aktuelle Zuweisung in eine spezialisierte Sprechstunde zeigt nun, dass sich eine schwere muskuläre Obstruktion subaortal ausgebildet hat, die die Hämodynamik der Fontan-Zirkulation kompromitiert. Innerhalb weniger Wochen findet eine Re-Operation statt.

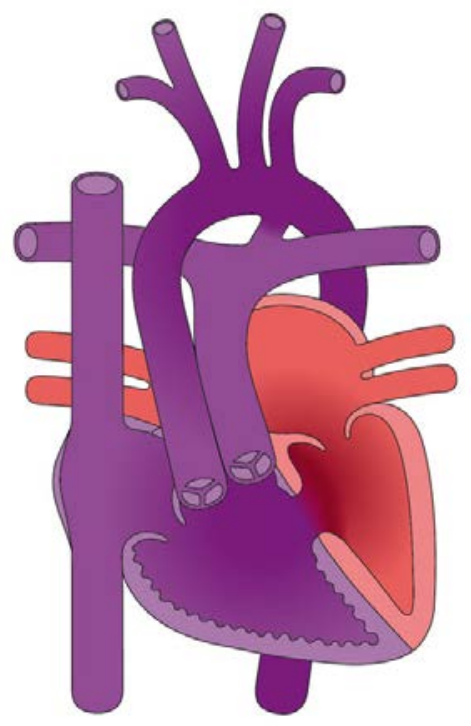

Abbildung aus: www.congenital-heart-disease.ch; New Media Center, University of Basel 
Es ist zu erwarten, dass eine kontinuierliche Nachbetreuung die Häufigkeit ernsthafter Komplikationen senkt und erlaubt, elektiv statt notfallmässig Eingriffe durchzuführen, die aus diesem Grunde wiederum mit einer geringeren Morbidität und Mortalität verbunden sind. Konkrete Daten diesbezüglich sind aber schwer zu erfassen und zurzeit ausstehend.

Eine adäquate Nachbetreuung im Erwachsenenalter lässt sich durch organisatorische und edukative Massnahmen erreichen.[9] Hier spielt das Konzept der Transition eine zentrale Bedeutung.

\section{Wozu Transition - Weitere Beispiele}

Jugendliche und junge Erwachsene mit chronischen Erkrankungen finden sich in allen Fachbereichen der Medizin, von der Endokrinologie über die Pneumologie hin zur Gastroenterologie oder Nephrologie.

In der Hämatologie ist beispielsweise die Hämophilie eine chronische Erkrankung, die von den Patienten viel Wissen und Fertigkeiten im Selbstmanagement verlangt. Dies beinhaltet Bereithaltung, Aufbereitung und Applikation des Faktorpräparats, aber auch ein angepasstes Freizeitverhalten. Nur so können Komplikationen von Blutungen in Gelenken, Muskel und Gehirn verhindert werden.

In der Infektiologie ist HIV im Jugendalter weltweit eine immer wichtigere Erkrankung. Während die Häufigkeit von neu angesteckten Kindern seit fünfzehn Jahren abnimmt, entwickelte sich HIV zur zweithäufigsten Todesursache im Jugendalter. Die Behandlung wird oft zu spät initiiert und antiretrovirale Toxizitäten treten in verschiedenen Organsystemen auf. Eine dauerhafte und langfristige Betreuung und Medikamenten-Adhärenz ist überlebensnotwendig.

\section{Definitionen}

Transitionen sind komplex und multidimensional. Ein Mensch durchläuft im Lauf seines Lebens verschiedene Transitionen. Es handelt sich dabei um unterschiedliche Arten von Veränderungen.[10]

\begin{tabular}{|l|l|}
\hline Entwicklungsbedingte Transition & Situationsbedingte Transition \\
Normale Veränderungen im Verlauf des & Veränderungen aufgrund von Umwelt, \\
Lebens & Kontext und sozialem Umfeld \\
z.B. Adoleszenz, Elternwerden, Alter & z.B. Wechsel in Ausbildung oder Beruf \\
\hline Gesundheits-/krankheitsbedingte & Organisationsbedingte Transition \\
Transition & Veränderungen in der Führung, Struktur \\
& oder Gesetzgebung einer Organisation \\
\hline
\end{tabular}


Published in final edited form as:

Pädiatrie up2date 2016; 11(01): 55-74 DOI: 10.1055/s-0041-107502

\begin{tabular}{|l|l|}
\hline Veränderung der gesundheitlichen & z.B. Sich wandelnde \\
Verfassung oder Betreuung & Gesundheitssysteme \\
z.B. Heimkehr nach Spitalaufenthalt, & \\
Anpassung an einer chronische & \\
Erkrankung & \\
\hline
\end{tabular}

Das Leben mit einer chronischen Erkrankung verursacht eine spezielle gesundheits/krankheitsbedingte Transition, auf die wir uns in diesem Artikel konzentrieren.

\section{Definition}

Transition beschreibt den Prozess, welcher Jugendliche und junge Erwachsene mit einer chronischen Erkrankung darauf vorbereitet, für Ihr Leben und ihre Gesundheit im Erwachsenenalter Verantwortung zu übernehmen. Es ist die Aufgabe der medizinischen Betreuungspersonen, den Jugendlichen im Prozess der Transition zu unterstützen.

Andere Arten von Transitionen können zeitgleich mit der hier definierten stattfinden. Von der Transition muss der Begriff des Transfers abgegrenzt werden. Oft wird dieser fälschlicherweise mit der Transition gleichgesetzt.

\section{Definition}

Transfer beschreibt den Moment, oder eine Serie von Momenten, in welchem die Betreuung der Jugendlichen und jungen Erwachsenen mit einer chronischen Erkrankung von der kinder- zur erwachsenenzentrierten Medizin wechselt.

\section{Die Herausforderung}

\section{Barrieren für eine erfolgreiche Transition}

Man spricht dann von einer gescheiterten Transition, wenn es vor oder nach dem Transfer in die Erwachsenenmedizin zu einem medizinisch nicht indizierten Betreuungsabbruch kommt oder die Therapieadhärenz des Jugendlichen abnimmt und letztendlich vermehrte Notfallsituationen eintreten [11]. Die Gründe für ein Scheitern können vielschichtig sein: sie lassen sich in der Organisation finden, in den Struktur- und Prozesskriterien, aber auch in den intrinsischen Aspekten der Adoleszenz.

\section{Didaktische Box 1}

Mögliche Gründe für eine gescheiterte Transition

Organisationale Aspekte

- Kein formaler Transitionsprozess

- Rigide institutionelle Transferstruktur 
Published in final edited form as:

Pädiatrie up2date 2016; 11(01): 55-74 DOI: 10.1055/s-0041-107502

- Beschränkung auf Altersgrenzen anstelle Entwicklungs-spezifischer Kriterien für die Einleitung der Transition: der Transitionsprozess wird zu spät oder zu früh eingeleitet.

- Ungenügende multidisziplinäre Zusammenarbeit; fehlende Kommunikation zwischen Kinder- und Erwachsenenmedizinern, unklare Zuständigkeiten im Transitionsprozesses, fehlendes gegenseitiges Verständnis für die unterschiedlichen Kulturen in der Kinder- und Erwachsenenmedizin

- Informations- und Wissensdefizit seitens der Familien/Patienten

- Langer Unterbruch in der medizinischen Betreuung zwischen dem Transfer von der Kinder- in die Erwachsenenmedizin

\section{Adoleszenten-spezifische Aspekte}

- Adoleszenz-bedingte kognitive, soziale und emotionale Veränderungen

- Mühe, das bekannte und familiäre System zu verlassen

- Ängste der Eltern

- Fehlende Ansprechpartner (Familie, Peers, Gesundheitspersonal)

- Fehlendes Interesse für Adoleszente seitens Kinder- und/oder Erwachsenenmediziner

- Zu früher Transfer, sich nicht bereit fühlen für die Erwachsenenmedizin

Aufgrund fehlender Prozess- und Strukturkriterien, wie zum Beispiel der Frage der Verantwortung für den Transitionsprozess, oder der Organisation finanzieller Vergütung wird meist nur auf den Transfer fokussiert und die Aussage „Transfer is common, Transition is rare" von Paul, M., et al. (2013) ist vielerorts immer noch zutreffend. Nicht selten besteht die Schwierigkeit einen Erwachsenenmediziner zu finden, der den Jugendlichen adäquat weiterbetreuen kann. Es ist Aufgabe des Kinderarztes oder pädiatrischen Facharztes, eine kompetente medizinische Nachbetreuung aufzugleisen. Ist der Erwachsenenmediziner, welcher die Behandlung des Patienten übernehmen wird bekannt, sollte dieser schriftlich über die Kranken- und Familiengeschichte informieren werden. Fehlende Informationen beim Erstbesuch auf der Erwachsenenseite können beim Jugendlichen und seiner Familie verständlicherweise Unsicherheit auslösen. Diese Ausgangslage erschwert dem Erwachsenenmediziner eine tragfähige Beziehung zum Patienten und seiner Familie aufzubauen [13]. Ebenfalls ein Hindernis kann ein früher Transfer auf die Erwachsenenmedizin darstellen. Dies kann Institutions-bedingt sein oder auf eine falsche Einschätzung der Transfer-Bereitschaft seitens des Kinderarztes zurückzuführen sein. Der Entwicklungsstand des Jugendlichen wird nicht selten überoder unterschätzt. Jugendliche selber geben an, dass sie sich oft noch zu jung fühlen, um in die Erwachsenenmedizin zu wechseln [14].

Merke:

Macht sich schon vor dem Transfer einen unregelmässiger Besuch der Sprechstunde bemerkbar, resultiert dies nach dem Transfer oft in einen „early drop-out". 
Published in final edited form as:

Pädiatrie up2date 2016; 11(01): 55-74 DOI: 10.1055/s-0041-107502

Nicht unterschätzt werden darf der kulturelle Unterschied zwischen der Kinder- und Erwachsenenmedizin. Auf der Kindermedizin wird vorwiegend ein familiäres System gelebt. Der Kinderarzt betreut den Patienten und dessen Familie über viele Jahre und es besteht ein enges Vertrauensverhältnis. Dieses aufzulösen ist nicht nur für die Familie schwierig, sondern auch für den behandelnden Arzt. Auf der Erwachsenenmedizin hingegen wird ein personenfokussiertes System gelebt und Selbstständigkeit und Eigenverantwortung wird erwartet [15]. Ein vertrautes Umfeld zu verlassen kann in beim Jugendlichen und in der Familie Angst auslösen. Seitens der Eltern bestehen zusätzlich Ängste, dass sich der Jugendliche nur ungenügend um seine Erkrankung kümmern wird.

Merke:

Die Transition sollte demzufolge nicht nur eine kontinuierliche Begleitung der Jugendlichen beinhalten, sondern auch der Familie.

Zudem fällt die Transition in eine Zeit, in der die Adhärenz an ein Therapieschema für den Adoleszenten grundsätzlich erschwert ist [16]. In dieser Entwicklungsphase werden Grenzen ausgelotet werden und Widerstand gesucht. Dazu kann auch die Auflehnung gegen medizinische Notwendigkeiten gehören.

\section{Elemente einer erfolgreichen Transition}

Das Ziel einer gelungenen Transition beschränkt sich nicht nur auf eine adäquate medizinische Betreuung, sondern beinhaltet auch die Förderung der Selbstmanagementkompetenzen. Damit dies gelingen kann, braucht es neben den Anpassungen der Organisation auch eine angepasste entwicklungsspezifische Begleitung [17]. Auch wenn bislang keine klare Evidenz über die Schlüsselelemente einer erfolgreichen Transition vorhanden ist, stimmen Patienten, Eltern und Experten über die wichtigsten Komponenten einer erfolgreichen Transition überein [18-20].

\section{Didaktische Box 2}

\section{Schlüsselelemente für eine erfolgreiche Transition}

- Absprache und partnerschaftliche Zusammenarbeit zwischen Kinder- und Erwachsenenarzt

- Früher Start des Transitionsprozesses auf pädiatrischer Seite (spätestens 1 Jahr vor dem Transfer)

- Individueller Transitionsplan

- Alter zum Zeitpunkt des Transfers (>17 Jahre)

- Schriftliche Übergabe

- Klar identifizierter Ansprechpartner auf der Erwachsenenseite

- Kontrolle ob Transfer stattgefunden hat

- Einverständnis und Unterstützung der Eltern

- Adoleszentenfreundliche Sprechstunden: Jugendlichen die Gelegenheit geben alleine mit dem Gesundheitspersonal zu sprechen, in den Konsultationen genügend Zeit für Fragen einplanen, kurze Wartezeiten 
Published in final edited form as:

Pädiatrie up2date 2016; 11(01): 55-74 DOI: 10.1055/s-0041-107502

Unabhängig von einem allfälligen Transfer auf die Erwachsenenmedizin sollte die Edukation und Förderung der Patientenpartizipation durch die Kindermediziner schon frühzeitig eingeleitet werden. Auch wenn die Transition Patient und Eltern betrifft, sollte doch darauf geachtet werden, dass der Jugendliche Zeit erhält, alleine mit dem Arzt oder einer anderen Gesundheitsperson über seine persönlichen Anliegen zu sprechen.

Merke:

Je nach kognitiver Entwicklung des Jugendlichen, sollte dem Jugendlichen ermöglicht werden, einen Teil der Sprechstunde alleine zu besuchen.

Bezüglich dem Zeitpunkt des Transfers hat sich ein höheres Alter bewährt: Neuere Studien empfehlen einen flexiblen Übertritt im Alter zwischen 18 und 21 Jahren, je nach kognitiver Entwicklung des Jugendlichen [14, 21]. Der einzige Vorteil starrer institutioneller Vorgaben ist, dass allen Beteiligten klar ist, dass ein Wechsel auf die Erwachsenenmedizin unausweichlich ist. Patient, Eltern und Kinderarzt können sich damit frühzeitig auf den Transfer einstellen und vorbereiten.

Merke:

Je älter der Patienten zum Zeitpunkt des Transfers ist, desto kleiner ist das Risiko für „early drop-out“

Da Verhaltensänderungen, wie z.B. die Übernahme von Eigenverantwortung, nicht von heute auf morgen umgesetzt werden können, empfiehlt es sich frühzeitig mit dem Transitionsprozess zu beginnen: idealerweise im Alter von 12 Jahren, spätestens jedoch ein Jahr vor dem Transfer $[18,21]$ Zur Strukturierung des Transitionsprozesses hat sich die Verwendung von Checklisten bewährt, welche individuell auf den Patienten angepasst werden können (siehe Transition-Tools) [22]. Die Erstellung eines schriftlichen Transitionsplan in Absprache mit der Familie hilft, die wichtigsten Meilensteine und den Transferzeitpunkt anzusprechen und festzuhalten.

Merke:

Bei der letzten Kontrolle beim Kinderarzt sollte geklärt werden, bei wem und wann die nächste Verlaufskontrolle stattfindet.

Wird der Patienten von einem multidisziplinären Team betreut, ist es wichtig, dass alle Beteiligten, zum Beispiel Physiotherapeuten, Ergotherapeuten oder Sozialberater, über die mit dem Patienten vereinbarten Meilensteine informiert sind. Am besten wird das Dokument in der Patientenakte abgelegt und beim Transfer inklusive der Zusammenfassung der Krankengeschichte an den Erwachsenenmediziner weitergeleitet. 
Die Zusammenfassung zuhanden des nachbetreuenden Arztes sollte Diagnose, Medikation, Krankheitsverlauf sowie wesentliche Informationen über den soziokulturellen Hintergrund enthalten.

\section{Transition aus Sicht der einzelnen Interessengruppen}

\section{Transition - eine Teamarbeit}

Betroffene mit chronischer Erkrankung werden in der Regel von verschiedenen medizinischen Diensten in ihrem Krankheitsprozess begleitet. Je nach Erkrankung sind neben Arzt und Pflege zusätzlich Ernährungsberater, Physiotherapeuten, Sozialarbeiter sowie weitere Dienste in den Behandlungsprozess involviert. Dem Hausarzt kommt oft eine Schlüsselrolle zu. Eltern und Familie sind naturgemäss weitere wichtige Bezugspersonen für Patienten mit chronischer Erkrankung. Um die Transition zwischen all diesen Beteiligten zu koordinieren, hat sich der Einsatz eines Transitionskoordinators bewährt, welcher den Patienten und seine Familie während des Transitionsprozesses begleitet und gemeinsam mit innen die einzelnen Schritte festlegt [23].

Merke: Transition verlangt einen multidisziplinären Ansatz. 


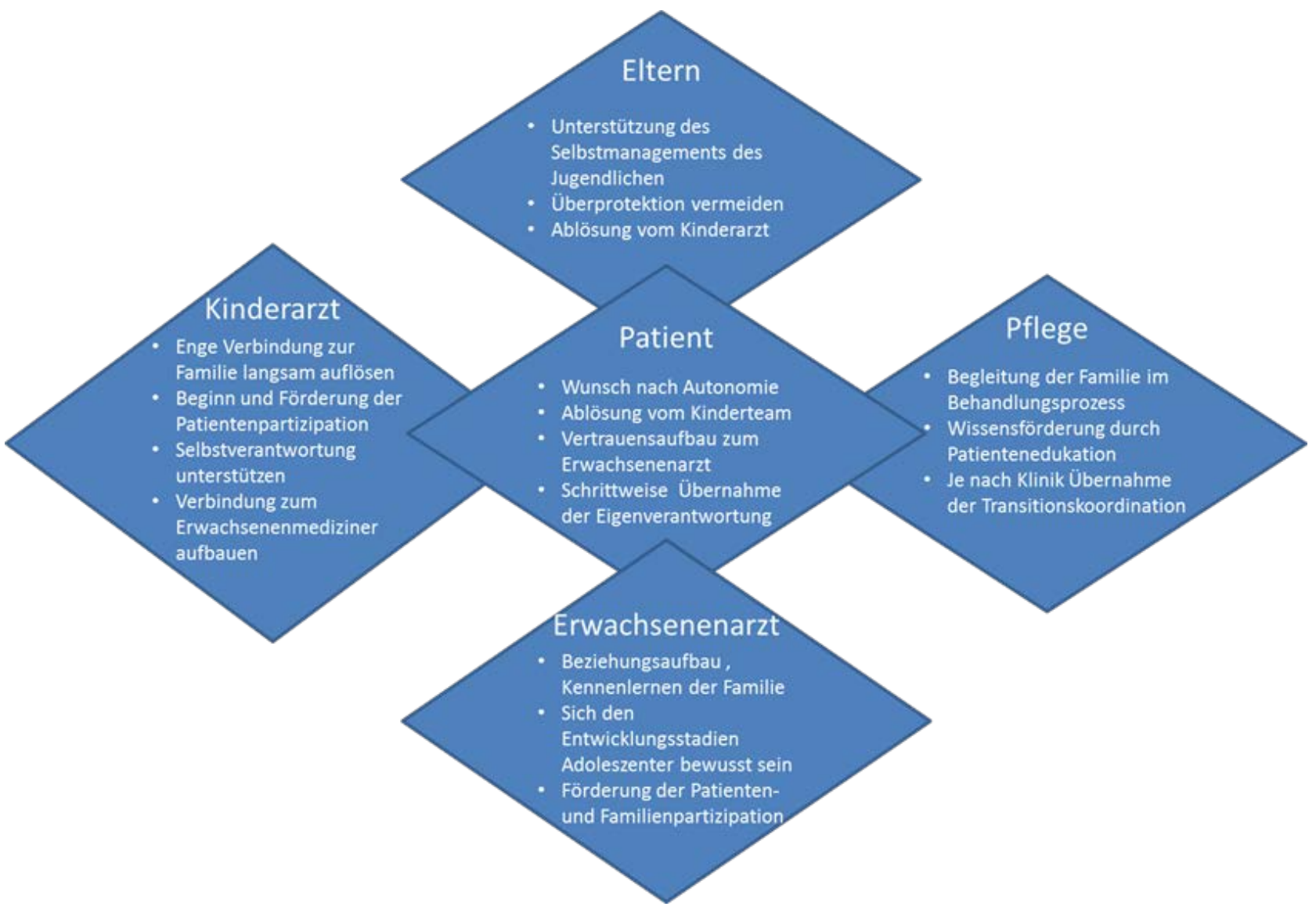

Grafik adaptiert nach Kovacs, A. \& McCrindle, B. So hard to say goodbye: transition from paediatric to adult cardiology care. Nature Reviews Cardiology, 2014; 11, 5162.

\section{Transitionskoordinator}

Zu den Hauptaufgaben eines Transitionskoordinators gehört die Schulung und Beratung des Patienten und seiner Familie im Transitionsprozess, Unterstützung und Förderung der Eigenverantwortung sowie die Vorbereitung und Planung des Transfers. Der Transitionskoordinator übernimmt die Koordination zwischen den einzelnen Diensten und hat eine wichtige Brückenfunktion zwischen der Kinder- und der Erwachsenenmedizin inne [24]. Diese Aufgabe kann von Pflegefachpersonen mit höheren Abschlüssen, aber auch von anderen Personen aus dem Gesundheitswesen übernommen werden, wie z.B. Sozialdiensten, Ärzten oder Psychologen.

\begin{tabular}{|l|l|}
\hline $\begin{array}{l}\text { Tabelle 2 } \\
\text { Aufgaben des Transitionskoordinators [24, 25] }\end{array}$ \\
\hline Vorbereitung & $\begin{array}{l}\text { - Familienanamnese und Selbstmanagement-Assessment } \\
\text { - Erarbeitung eines individuellen Transitionsplan mit der Familie }\end{array}$ \\
\hline Begleitung & $\begin{array}{l}\text { - Ansprechpartner für den Jugendlichen und seine Familie } \\
\text { während dem Transitionsprozess } \\
\text { - Kontinuierliche Patientenedukation }\end{array}$ \\
\hline
\end{tabular}


Published in final edited form as:

Pädiatrie up2date 2016; 11(01): 55-74 DOI: 10.1055/s-0041-107502

\begin{tabular}{|l|l|}
\hline & $\begin{array}{l}\text { - Begleitung der Familie in der Verantwortungsübergabe } \\
\text { - Vorbereitung auf die Erwachsenenmedizin }\end{array}$ \\
\hline $\begin{array}{l}\text { Behandlungs- } \\
\text { koordination }\end{array}$ & $\begin{array}{l}\text { - Nimmt Rücksprache mit den im Behandlungsprozess } \\
\text { beteiligten Diensten }\end{array}$ \\
& $\begin{array}{l}\text { - Ermöglicht allen Beteiligten Einblick in den Transitionsprozess } \\
\text { - Organisiert, wenn möglich das persönliche Kennenlernen des } \\
\text { Erwachsenenteams }\end{array}$ \\
& $\begin{array}{l}\text { - Bietet dem Behandlungsteam Unterstützung zu adoleszenten- } \\
\text { spezifischen Fragen }\end{array}$ \\
\hline Auswertung & $\begin{array}{l}\text { - Überprüft, ob der Transitionsprozess für den Jugendlichen } \\
\text { und seine Familie erfolgreich verlief }\end{array}$ \\
& $\begin{array}{l}\text { Abschluss und Auswertung des Transitionsprozesses mit } \\
\text { dem Jugendlichen und seiner Familie }\end{array}$ \\
\hline
\end{tabular}

\section{Perspektive des Patienten}

Zu den Schlüsselkomponenten des Erwachsenwerdens gehört Verantwortung zu übernehmen und Entscheidungen zu treffen. Jugendliche wünschen sich zunehmend Unabhängigkeit von den Eltern und dem Gesundheitspersonal. Freunde werden immer wichtiger und neue Partnerschaften bilden sich. Dies alles kann auch Auswirkungen auf die Adhärenz des Jugendlichen haben, was dazu führt, dass Medikamente abgelehnt werden, der Therapieplan nicht mehr befolgt wird [26]. Erschwerend kommt hinzu, dass Jugendliche oft nur ungenügend über ihre Diagnose informiert sind und die Auswirkungen ihres Handelns auf die Erkrankung nur ungenügend einschätzen können. Dies alles führt nicht selten zu einer Ablehnung von Folgeuntersuchungen. Eltern und Gesundheitspersonal sind in dieser Situation stark gefordert.

\section{Perspektive der Eltern}

Eltern spielen eine zentrale Rolle im Transitionsprozess. Sie haben bis anhin die Verantwortung für das Behandlungs- und Therapiemanagement übernommen. Auch die Eltern haben über die Jahre hinweg eine enge Verbindung zum Kinderarzt aufgebaut. Den Kinderarzt verlassen zu müssen löst deshalb auch bei einigen Eltern Angst aus. Nicht selten äussern sie die Sorge, in der Erwachsenenklinik nicht mehr erwünscht zu sein und haben zudem den Eindruck, dass sich ihr Jugendlicher nicht genügend um seine Erkrankung kümmert. Das Gefühl von der Behandlung ausgeschlossen zu werden, kann solche Sorge verstärken. Die regelmässigen Kontrollen beim Kinderarzt sind für die Eltern ebenfalls eine Möglichkeit, ihr Informationsbedürfnis zu stillen und Sicherheit im Umgang mit dem Jugendlichen zu erlangen. Ziel und Inhalte der Transition sollten deshalb auch mit den Eltern besprochen werden. Es hat sich gezeigt, dass eine gute Unterstützung der Eltern einen positiven Einfluss auf das psychologische Wohlbefinden des Jugendlichen hat [5]. Somit ist es wichtig, die Eltern frühzeitig in den Prozess der Verantwortungsübergabe an ihr Kind einzubinden. 
Published in final edited form as:

Pädiatrie up2date 2016; 11(01): 55-74 DOI: 10.1055/s-0041-107502

\section{Perspektive der Kindermedizin}

In gleichem Masse wie Eltern und Patienten eine langjährige Beziehung zur Kindermedizin aufbaut haben, haben Kinderärzte und Pflegende über die Jahre ein Bande zur Familie und zum Patienten geknüpft. Kindermediziner bekunden teilweise Mühe ihre Jugendlichen gehen zu lassen und machen sich Sorgen, ob diese auf der Erwachsenenmedizin genügend betreut werden.

In unserer Erfahrung haben Kinderärzte die Tendenz die Langzeitfolgen chronischer Erkrankungen im Erwachsenenalter unzureichend wahrzunehmen. Dies führt zu einer eher optimistischen Grundhaltung, die zeitweise mit einer eher skeptischen Grundhaltung der Erwachsenenmediziner in Kontrast steht [26]. Dies löst je nach Situation bei der Familie ein Gefühl der Unsicherheit aus und erschwert die Beziehungsaufnahme durch den Erwachsenenmediziner. Pflegefachpersonen können hier eine zentrale Rolle in der Beratung und Begleitung der Jugendlichen und ihren Familien übernehmen und als neutrale Ansprechpartner das Bindeglied zwischen Kinder- und Erwachsenenmedizin übernehmen.

\section{Perspektive der Erwachsenenmedizin}

Die Transition endet nicht mit dem Transfer in die Erwachsenenmedizin. Erwachsenenmediziner und Pflegende haben die wichtige Aufgabe den Transitionsprozess weiterzuführen und abzuschliessen. Hierbei spielt der Informationsaustausch zwischen Kinder- und Erwachsenenmedizin eine grosse Rolle. Ein guter Austausch ermöglicht Pflegenden und Ärzten nahtlos patientenrelevante Themen weiterzuführen und adoleszenten-spezifische Aspekte auch auf der Erwachsenenmedizin zu berücksichtigen. Bei komplexen Erkrankungen, wie bei angeborenen Herzfehlern, bleibt dem Erwachsenenteam oft nur wenig Zeit, ein Vertrauensverhältnis zum Patienten aufzubauen. Bereits kurz nach dem Transfer müssen Folgen des Herzfehlers besprochen werden oder neue Aspekte, wie z.B. Familienplanung und Schwangerschaft werden relevant. Dies kann die Beziehung zwischen Patient, Familie und den Erwachsenenmediziner vor eine grosse Herausforderung stellen. Rasch werden Jugendliche wie Erwachsene behandelt und adoleszenten-spezifische Besonderheiten ignoriert. Gefordert sind Erwachsenenmediziner auch im Umgang mit den Eltern. Einen Umgang zu finden, in dem die Sichtweise der Eltern mit einbezogen wird, der Jugendliche aber trotzdem genügend Raum erhält um seine Autonomie zu entfalten und Verantwortung für seine Erkrankung zu übernehmen, stellt viele Erwachsenenmediziner vor eine grosse Herausforderung.

Merke: Eine gute Möglichkeit für Personen im Gesundheitswesen sich mit dem Thema Adoleszenz und Transition auseinanderzusetzen bietet die 1999 gegründete Arbeitsgruppe EuTEACH (European Training in Effective Adolescent Care and Health). Weitere Informationen zu Angebot und Ausbildung findet man unter www.euteach.com 
Published in final edited form as:

Pädiatrie up2date 2016; 11(01): 55-74 DOI: 10.1055/s-0041-107502

\section{Richtlinien und Empfehlungen zur Transition}

\section{Krankheits-unspezifische Empfehlungen}

Mit dem Ziel, allen Ärzten - ob nun Grundversorger oder Spezialisten - die Jugendliche mit chronischen Erkrankungen behandeln die minimalen Anforderungen einer erfolgreichen Transition aufzuzeichnen, wurden in Amerika im Jahre 2002 erstmals entsprechende Richtlinien publiziert. Diese Richtlinien halten fest:[27]

- Es ist zu gewährleisten, dass Jugendliche mit chronischen Erkrankungen zu jeder Zeit ihren ärztlichen Ansprechpartner kennen, der eine adäquate Betreuung sicher stellen kann.

- Kernkompetenzen und Fähigkeiten für eine alters-gerechte Betreuung müssen definiert und in die entsprechende ärztliche Ausbildung aufgenommen werden.

- Die wesentlichen medizinischen Informationen aus der Kindheit müssen an die nachbehandelnden Ärzte weiter geleitet werden.

- In Zusammenarbeit mit dem Jugendlichen und seiner Familie wird ein schriftlicher Transitionsplan verfasst.

- Es ist sicher zu stellen, dass jederzeit ein ausreichender Versicherungsschutz vorhanden ist, der ebenfalls die Kosten abdeckt die durch den Transitionsprozess selbst entstehen.

Diese ersten, mehr organisatorischen Schritte im Transitionsprozess werden ergänzt durch patienten-zentrierte Gespräche mit Wissensvermittlung, die die Jugendlichen konkret auf den Übertritt in das Erwachsenenleben begleiten. Hier wiederum wird Wert darauf gelegt, dass

- die Gespräche zukunfts-orientiert und flexibel gehalten werden

- ein Betreuungswechsel angesprochen ist

- zu persönlicher und medizinische Selbständigkeit motiviert wird

- der Transitionsplan detailliert besprochen wird

- die Transition als positives Erlebnis gefeiert wird.[28]

Sieben Jahre nach der Publikation dieser Richtlinien wird ernüchtert festgestellt, dass lediglich 2 von 5 Jugendlichen die einer Transition bedürfen, einen Prozess erleben, der die obigen minimalen Anforderungen erfüllt.[29] Defizite in der Umsetzung der Transition sind primär auf ein strukturelles Problem auf Seiten der Gesundheitsversorger und weniger auf die fehlende Bereitschaft der Jugendlichen zurückzuführen. 
Published in final edited form as:

Pädiatrie up2date 2016; 11(01): 55-74 DOI: 10.1055/s-0041-107502

2011 publizierte die American Heart Association ein krankheits-spezifisches Konzept, wie die Transition bei Adoleszenten mit AHF strukturiert werden sollte.[13] Die Publikation bewertete die vorhandene Evidenz der Empfehlungen gemäss dem zu erwartenden Nutzen (Klasse I: hoch, Klasse II: höher als möglicher Schaden, Klasse III: schädlich) und der Qualität der zu Grunde liegenden Studien (A: hohe Qualität, Meta-Analysen oder verschiedene randomisierte Studien, B: einzelne randomisierte Studie, C: Expertenmeinung). Sämtliche Empfehlungen wurden als IC eingestuft, d.h. sie basierten auf Expertenmeinung und es wurde ausschliesslich ein Nutzen postuliert. Die Monotonie der Empfehlungsgrade bringt zum Ausdruck, wie dünn weiterhin die wissenschaftliche und methodologische Basis ist, auf der das Konzept der Transition fusst.

In Ergänzung zu den allgemeinen Empfehlung der amerikanischen Gesellschaft für Pädiatrie[27] halten die krankheits-spezifischen Richtlinien fest, dass

- dem Hausarzt/Kinderarzt die Rolle des primären Gesundheitsversorgers zukommt, der im Zentrum der patienten-orientierten medizinischen Versorgung steht

- medizinische Abklärungen durch entsprechend ausgebildete Kardiologen durchgeführt werden sollten

- regelmässige Verlaufskontrollen unablässlich sind.

Diese Empfehlungen beschränken sich nicht auf AHF sondern können stellvertretend für alle Erkrankungen aufgefasst werden, bei denen hoch-spezialisierte Medizin eingesetzt wird.

Die Empfehlungen gehen im Weiteren auf Krankheits-spezifische Aspekte ein und betonen die Wichtigkeit des rechtzeitigen, d.h. bereits in der frühen Adoleszenz, Ansprechens verschiedener Themenblöcke. Diese umfassen die Aspekte der Sexualität, Verhütung und Vererbbarkeit des Herzfehlers, der körperlichen Belastung inklusive Empfehlungen bezüglich Sport, Freizeit und Beruf, der Berufswahl und Unterstützung in der Ausbildung, sowie der generellen Wissensvermittlung bezüglich des Herzfehlers und seiner Folgen.

Diese Empfehlungen beruhen auf einem Modell, das Transition als eine Einbahn aus einem kinder-zentrierten medizinischen Umfeld in eine Erwachsenen-orientierte Umgebung versteht. Beide Umgebungen sind eigenständige medizinische Systeme. Der Zeitpunkt wie auch der Umsetzungsrhythmus der Transition werden in der Pädiatrie geplant und eingeleitet, das adulte Umfeld ist der passive Empfänger neuer Patienten. Die Wirklichkeit sieht anders aus. Transition ist ein dynamischer Prozess, auf den unterschiedliche Kräfte einwirken, seitens der Familie, des Patienten und seines Umfeldes, seitens der Gegebenheiten in der Gesundheitsversorgung. Je nach Situation kann der Prozess beschleunigt oder gebremst werden und idealerweise ist es die Interaktion der einzelnen Player im Transitionsprozess, die die Geschwindigkeit des Prozesses vorgibt. 


\section{Vor- und Nachteile verschiedener Transitionskonzepte}

Aus ärztlicher Sicht gibt es verschiedene Möglichkeiten, wie eine kontinuierliche Betreuung über das Adoleszenten-Alter hinweg erreicht werden kann. Diesen Gegebenheiten haben sich die Transitions- und Betreuungskonzepte anzupassen. Am Beispiel angeborener Herzfehler lässt sich dies exemplarisch darstellen.

Die Betreuung kann sich in folgenden Umfeldern abspielen:

- Kinderkardiologie

- Allgemeine Erwachsenenkardiologie

- Kinder- oder Erwachsenenkardiologie mit Zusatzausbildung

Unter Zusatzausbildung wird verstanden, dass sich Kinderkardiologen auf dem Gebiet der Erwachsenenkardiologie weiter ausbilden (und umgekehrt), damit die Betreuung in allen Lebensphasen bestmöglichst gewährleistet ist. Die jeweiligen Vorund Nachteile sowie die Herausforderungen der verschiedenen Versorungskonzepte sind in Tabelle 3 aufgeführt. Wichtig ist zu betonen, dass ein Transitionskonzept unabhängig von der weiterführenden Betreuung erstellt werden sollte.

Merke:

Die Transition beschränkt sich nicht nur auf den Transfer und das Sicherstellen einer ununterbrochenen Betreuung, sondern soll vielmehr Adoleszente darauf vorbereiten Eigenverantwortung für ihre chronische Erkrankung in einem Erwachsenen-orientierten Gesundheitssystem zu übernehmen. 
Published in final edited form as:

Pädiatrie up2date 2016; 11(01): 55-74 DOI: 10.1055/s-0041-107502

Tabelle 3: Betreuungskonzepte transitions-bedürftiger Adoleszenter

\begin{tabular}{|c|c|c|c|}
\hline & Vorteile & Nachteile & Herausforderungen \\
\hline $\begin{array}{l}\text { Lebenslange Betreuung } \\
\text { durch Kinderärzte }\end{array}$ & $\begin{array}{l}\text { - Kontinuierliche persönliche Arzt- } \\
\text { Patienten-Beziehung } \\
\text { - Kein Informationsverlust } \\
\text { - Kleines Risiko eines Betreuungs- } \\
\text { unterbruches } \\
\text { - Das direkte Erheben von Outcome- } \\
\text { Ergebnissen im Erwachsenenalter } \\
\text { kann Änderungen von } \\
\text { Behandlungspfaden/-methoden im } \\
\text { Kindesalter einfacher beeinflussen }\end{array}$ & $\begin{array}{l}\text { - Beschränkte Expertise für } \\
\text { erworbene Erkrankungen im } \\
\text { Erwachsenenalter (z.B. die koronare } \\
\text { Herzkrankheit) } \\
\text { - Hospitalisation als Erwachsener im } \\
\text { Kinderkrankenhaus } \\
\text { - Fehlender Informationshintergrund } \\
\text { bei Hospitalisation im adulten } \\
\text { Krankenhaus } \\
\text { - Kein "frischer" Blick auf die } \\
\text { medizinische Situation zum } \\
\text { Zeitpunkt des Transfers } \\
\text { - Zurückhaltung bei Themen } \\
\text { bezüglich Schwangerschaft / } \\
\text { Sexualität seitens der Kinderärzte }\end{array}$ & $\begin{array}{l}\text { - Arzt und Patient altern gemeinsam; } \\
\text { beidseitige Anerkennung des } \\
\text { Rollenwechsel ist nötig (vom } \\
\text { Kinderarzt zum Ansprechpartner } \\
\text { für Fragen im Erwachsenenalter / } \\
\text { vom pädiatrischen Patienten zum } \\
\text { adulten Patienten mit } \\
\text { Eigenverantwortung) }\end{array}$ \\
\hline $\begin{array}{l}\text { Gemeinsame Betreuung } \\
\text { durch Kinderärzte und } \\
\text { Erwachsenenärzte (ohne } \\
\text { jeweilige zusätzliche } \\
\text { Spezialisierung) }\end{array}$ & $\begin{array}{l}\text { - Betreuung durch sowohl pädiatrisch } \\
\text { als auch adulte Spezialisten } \\
\text { gewährleistet. } \\
\text { - Kein Informationsverlust } \\
\text { - Kleines Risiko eines Betreuungs- } \\
\text { unterbruches }\end{array}$ & $\begin{array}{l}\text { - Personalressourcen-Bedarf (zwei } \\
\text { Spezialisten pro Patient nötig) und } \\
\text { hoher Planungsbedarf } \\
\text { - Sicherstellung einer gemeinsamen } \\
\text { Betreuung im Rahmen der } \\
\text { Hospitalisation erschwert }\end{array}$ & $\begin{array}{l}\text { - Klärungen der Verantwortungen und } \\
\text { der führenden Rolle } \\
\text { - Beidseitige Bereitschaft zur } \\
\text { konstruktiven Zusammenarbeit nötig } \\
\text { - Praktische Anwendung im } \\
\text { hektischen Berufsalltag }\end{array}$ \\
\hline $\begin{array}{l}\text { Übergabe der Betreuung vom } \\
\text { Kinderarzt an einen } \\
\text { spezialisierten } \\
\text { Erwachsenenarzt (oder } \\
\text { Betreuung durch einen } \\
\text { Kinderarzt mit } \\
\text { Zusatzausbildung in } \\
\text { Erwachsenenmedizin) }\end{array}$ & $\begin{array}{l}\text { - Zu jeder Zeit optimale Betreuung } \\
\text { durch Spezialisten } \\
\text { - Der Übergang vom pädiatrischen } \\
\text { ins erwachsenen } \\
\text { Gesundheitsumfeld wird durch } \\
\text { einen Ärzte- und Klinikwechsel } \\
\text { markiert } \\
\text { - Teamarbeit wird nach aussen } \\
\text { sichtbar gemacht }\end{array}$ & $\begin{array}{l}\text { - Patienten und deren Familien } \\
\text { müssen sich an einen "neuen" Arzt } \\
\text { gewöhnen } \\
\text { - Risiko eines Betreuungs- } \\
\text { Unterbruches }\end{array}$ & $\begin{array}{l}\text { - Strukturierte Zusammenarbeit } \\
\text { zwischen Kinder- und } \\
\text { Erwachsenenmedizin nötig } \\
\text { - Definierte Ansprechpartner müssen } \\
\text { bekannt sein }\end{array}$ \\
\hline
\end{tabular}


Published in final edited form as:

Pädiatrie up2date 2016; 11(01): 55-74 DOI: 10.1055/s-0041-107502 


\section{Neue Strukturen zur Transition von Menschen mit Behinderung}

Jugendliche und junge Erwachsene mit Behinderung haben bisher zu wenig von Transitionskonzepten profitiert. Insbesondere Menschen mit einer geistigen oder mehrfachen Behinderung sind oft unterversorgt. Vielen Ärzten fehlen barrierefreie Räumlichkeiten und spezielle Kenntnisse zu dieser Patientengruppe. Die Folge sind verspätete Diagnosen und fehlerhafte Behandlungen.

Neue gesetzliche Grundlagen ermöglichen in Deutschland die Entwicklung von Medizinischen Behandlungszentren für Erwachsene mit Behinderung (MZEB). MZEB sind spezialisierte ambulante Einrichtungen und ergänzen die hausärztliche und fachärztlichen Versorgung für Menschen, die aufgrund der Art, Schwere oder Komplexität der Behinderung eine spezielle Expertise benötigen. An MZEB soll eine optimale Versorgung geplant werden, damit die Betroffenen ein möglichst selbstständiges und selbstbestimmtes Leben führen können. Ein zentrales Ziel der MZEB ist die Versorgungskontinuität für junge Erwachsene mit Behinderung sicherzustellen. Dafür soll eine systematische Transition organisiert werden, damit der Übergang von der kinder- zur erwachsenenzentrierten Versorgung klappt.

Am Evangelischen Krankenhaus Alsterdorf in Hamburg wurde ein MZEB als bundesweites Modellprojekt im Sommer 2015 eröffnet.

\section{Inhalte der Transition}

Thematisch dreht sich die Transition um all jene Aspekte, die dem Jugendlichen ermöglichen, für seine Gesundheit im Erwachsenenalter Verantwortung zu übernehmen, beziehungsweise, die dem Jugendlichen diesen Prozess erschweren. Es geht dabei um medizinisches Wissen zu erkrankten Organen und Medikamenten, Fertilität und Verhütung, Freizeitaktivitäten, gesunde Ernährung, Ausbildung und Beruf, Versicherungen, Noxen wie Alkohol und Nikotin, und psychosoziale Themen wie Körperbild und Sexualität.

Beispielhaft hier eine Checkliste, wie sie am Zentrum für angeborene Herzfehler am Inselspital Bern verwendet wird. Sie erlaubt, einen Überblick über die behandelten Themen zu behalten und dient der Dokumentation des Transitions-Fortschrittes. Da sich der Transitionsprozess über mehrere Jahre erstreckt, ist es oft schwer den entsprechenden Überblick zu behalten. Die Themen lassen sich je nach Krankheitsbild anpassen. (Checkliste)

\section{Bedürfnisse Adoleszenter}

Die Transition findet in einer Zeit grosser physiologischer Änderungen und Prozesse statt. Jugendliche werden als unzuverlässig, launisch und gewaltsam beschrieben. Sie durchlaufen Meilensteine in Bereichen der

\section{- Autonomie}


Published in final edited form as:

Pädiatrie up2date 2016; 11(01): 55-74 DOI: 10.1055/s-0041-107502

- Beziehungsbildung

- Körperwahrnehmung

- Identitätssuche

- Sexualität

- Kognition

- Bildung

Sie wollen keinen Arzt sehen - obwohl sie sich für ihre Gesundheit interessieren, was aus Unmengen an Einträgen und Diskussionen in Onlineforen klar wird. Warum verhalten sie sich manchmal so „irrwitzig“? Ihr Verhalten ist ein Produkt aus Hirnentwicklung, hormonellen Änderungen, eigenen Wünschen und Interessen, Medien, sozialen Normen, und dem Verhalten der Peergruppe und Eltern. Das limbische System verstärkt während der Pubertät Emotionen und Arousal durch eine veränderte Ausschüttung von Dopamin. Der präfrontale Kortex wird erst gegen Ende der Pubertät effizient in Impulskontrolle und Planungsdenken.

Um der Unwegsamkeit der Jugend Rechnung zu tragen, wurden Konzepte entwickelt, die Schwelle der Inanspruchnahme medizinischer Dienstleistungen zu senken. Ein vielversprechender Ansatz sind sogenannte „Walk-in“-Sprechstunden. Dies bezeichnet eine während definierten Zeitfenstern „offene“ Sprechstunde, die Jugendliche ohne vorherige Anmeldung besuchen können. Sie müssen dadurch keine Gründe des Besuchs angeben, die womöglich den wahren Grund nicht umfassen. Sie sind auch flexibler in der Planung des Besuchs und nehmen selbstbestimmt die medizinische Versorgung in ihre Hände.[30]

Strukturell und inhaltlich scheinen folgende zwei Punkte am wichtigsten für eine erfolgreiche, adoleszenten-freundliche Dienstleistung zu sein: Sich Zeit nehmen für ein Gespräch mit dem Jugendlichen unter vier Augen, und die Zusicherung der Vertraulichkeit an den Jugendlichen.

Um die psychosoziale Entwicklung der Jugendlichen zu erfassen, hat sich der Anamnese-Fragebogen HEADSSS bewährt. Er deckt die enorme Breite an Themen $a b$, die während der Adoleszenz wichtig werden. Selbstverständlich steht dem Arzt frei, bestimmte Bereiche zu vertiefen oder wegzulassen. Ein Vorzug von HEADSSS ist das natürliche Fortschreiten des Gesprächs aus erwarteten, einfachen Fragen hin zu persönlichen Fragen. Dies gibt dem Arzt die Möglichkeit, Vertrauen aufzubauen.

\begin{tabular}{|l|l|}
\hline \multicolumn{2}{|l|}{ Tabelle $\mathbf{4}$} \\
H.E.A.D.S.S.S. Anamnese Fragebogen \\
\hline Home \& Environment & Wohnsituation, Familie \\
\hline Education \& Employment & Schule, Ausbildung, sich in der Klasse wohlfühlen \\
\hline Activities & Freizeitgestaltung, Sport \\
\hline Drugs & Konsum von Alkohol, Zigaretten, illegale Drogen \\
\hline
\end{tabular}


Published in final edited form as:

Pädiatrie up2date 2016; 11(01): 55-74 DOI: 10.1055/s-0041-107502

\begin{tabular}{|l|l|}
\hline Sexuality & $\begin{array}{l}\text { Freundschaften, Beziehungsaufbau, } \\
\text { Identitätsentwicklung, Körperbild }\end{array}$ \\
\hline Security & Sicherheitsverhalten, Unfallrisiko \\
\hline Suicide/Depression & Befinden, Stimmung, Sorgen, suizidales Verhalten \\
\hline $\begin{array}{l}\text { Adaptiert nach Goldenring, JM.; Rosen, DS. Getting into adolescent heads: an } \\
\text { essential update. Contemporary Pediatrics, 2004; 21(1): 64-90. }\end{array}$ \\
\hline
\end{tabular}

\section{Informationsmaterial}

Die Wissensvermittlung im Transitionsprozesses wird durch Informationsmaterial erleichtert. Unsere eigenen Erfahrungen zeigen, dass sich verschiedene Möglichkeiten anerbieten, den Jugendlichen Transitions-spezifische Inhalte zu vermitteln.

- Webseiten und Passports: Es lassen sich die Mitarbeiter vorstellen, der Ablauf des Transitions-Prozesses, sowie z.B. auch die Räumlichkeiten in denen sich der Prozess abspielt (siehe beispielsweise www.sickkids.ca/good2go). Dies gibt den Jugendlichen einen ersten konkreten Eindruck wie die weitere Betreuung auf der Erwachsenenseite aussehen wird. Zudem können Webseiten für die Wissensvermittlung genutzt werden und interaktiv durch Fragen das Wissen getestet werden. In der Kinderonkologie entstanden in den letzten Jahren „Passport"-Webseiten, die mittels persönlichem Login eine Online-Akte freischalten, die über Therapien, Risiken und Prävention von Spätfolgen informiert (siehe beispielsweise http://encca.cineca.org/) Der Passport kann auch ausgedruckt und unterschrieben dem Survivor angegeben werden. Der zeitliche Aufwand für das Erstellen hochwertiger Webseiten ist beträchtlich, nebst den anfallenden Kosten für den Unterhalt. (Abbildung)

- Informationsbroschüren: Für komplexe medizinische Themen (wie z.B. das Thema Endokarditis-Prophylaxe; Abbildung) die keinen raschen Änderungen des Wissens-Standes unterworfen sind, lässt sich in Broschüren das wesentliche patientengerecht zusammenfassen. Sie vereinfachen das direkte Gespräch und können zudem für Werbezwecke verwendet werden.

- Smart-Phone Apps: In der Schweiz sind über 85\% aller Jugendlichen im Besitze eines App-tauglichen Smartphones. Kommunikation bei Jugendlichen und Adoleszenten spielt sich vermehrt über Smart-phone basierte Kurznachrichten ab, wie z.B. das Kommunikations-App WhatsApp. Jugendliche schätzen es, wenn sie auf vertrauten Kommunikationskanälen mit ihren behandelnden Gesundheitsfachleuten in Kontakt treten können, Fragen stellen oder Termine verschieben können. Smart-Phones bieten zudem die Möglichkeit, Jugendlichen speziell entworfene Apps zu ihrem Krankheitsbild zu vermitteln, die innen als Informationsbasis dienen können. Im Gegensatz zu gedruckten Materialen haben Jugendliche ihr Smart-Phone immer auf sich, ob in Ferien oder zu Hause, und können somit einfach auf im App hinterlegte 
Published in final edited form as:

Pädiatrie up2date 2016; 11(01): 55-74 DOI: 10.1055/s-0041-107502

Informationen zurückgreifen. Im Bereich der angeborenen Herzfehler gibt es mittlerweile Apps die Herzfehler und die durchgeführten Eingriffe in dreidimensionalen Abbildungen erklären oder Apps die entwickelt wurden um Jugendliche über ihre Diagnosen, Medikamente und Herzfehler-spezifische Verhaltensmassnahmen zu informieren (Herz Kompass; Abbildung). Vorteile von Apps sind ihre vielseitige Nutzbarkeit und ihre modernes Erscheinungsbild, das Jugendliche anspricht. Nachteile sind ihre hohen Entwicklungskosten und die Notwendigkeit verschiedene Betriebssysteme abzubilden (iOS, Android) mit einem hohen Bedarf an Betriebsunterhalb.

\section{Forschung}

Es besteht in der Fachliteratur Einigkeit über die Wichtigkeit des Prozesses der Transition. Seit rund 10 Jahren ist die Anzahl Publikationen pro Jahr zum Thema Transfer und Transition angestiegen. Empirisch wurden bisher vor allem drei Themenbereiche der Transition erforscht:

1. Die Transitionsbereitschaft (engl. Transition readiness): der Wunsch und die Fähigkeit des Jugendlichen, unabhängig und verantwortungsbewusst für seine Gesundheit zu sorgen. Es wurde ihr Wissen und Selbstmanagement evaluiert, wobei das als „ausreichend“ erachtete Level in verschiedenen Krankheitsgruppen und Forschungsprojekten sehr unterschiedlich war.

2. Die Effektivität von Transitionsprogrammen. Hierbei konzentrierten sich die Forscher auf Prozess-Indikatoren (z.B. Anzahl Erwachsene, die Termine in der Transitions-Sprechstunde nicht wahrnehmen) und erkrankungsspezifische biochemische Outcomes (z.B. HbA1c, Kreatinin). Diese Indikatoren messen vor allem den medizinisch-biologischen Teil der Transition und vernachlässigen psychologische und soziale Aspekte. Langzeitmorbidität und -mortalität fehlen als Indikatoren, da die Kohorte überlebender und untersuchter Patienten noch jung ist. In der kinderonkologischen Nachsorge-Studien ist beispielsweise noch unklar, wie es Survivors im Alter von 50 oder 60 Jahren geht.

3. Die Erfahrung der Patienten. In qualitativen Studien zeigte sich, dass der Wechsel von der kinder- zur erwachsenenzentrierten Medizin für viele Patienten ein Kulturschock ist. Sie betonen, wie unbefriedigend ein plötzlicher Wechsel ist. Bei einer mangelhaften Vorbereitung fühlten sie sich oft deplatziert und überflüssig am neuen Spital oder in der neuen Praxis, was zu Betreuungsabbrüchen führte. Ihr aktiver Einbezug in die Entscheidungsprozesse war essentiell.

Allerdings dominieren bei bisherigen Publikationen Kohortenstudien, Fall-KontrollStudien und Fallbeschreibungen. Selten wurden quasi-experimentelle Interventionen beschrieben. Insgesamt bestehen noch grosse Lücken in unserem Wissen. Es ist 
Published in final edited form as:

Pädiatrie up2date 2016; 11(01): 55-74 DOI: 10.1055/s-0041-107502

aus wissenschaftlicher Sicht nicht klar, inwiefern wir mit den momentanen eingesetzten Modellen und Inhalten die Gesundheit und Lebensqualität von chronisch erkrankten Jugendlichen tatsächlich verbessern.

\section{Übersicht}

Lücken im wissenschaftlichen Wissen zu Transition

- Keine gut validierten Fragebögen, welche die „Transitionsbereitschaft“ messen

- Fehlende standardisierte Prozess-Indikatoren

- Fehlende Studien zu Langzeit-Outcomes

- Fehlende Kosten-Effektivitäts-Analysen

- Keine gut untersuchte Effektivität unterschiedlicher Transitionsmodelle

- Insgesamt fehlende randomisierte Interventionsstudien 
Published in final edited form as:

Pädiatrie up2date 2016; 11(01): 55-74 DOI: 10.1055/s-0041-107502

Abbildungen:

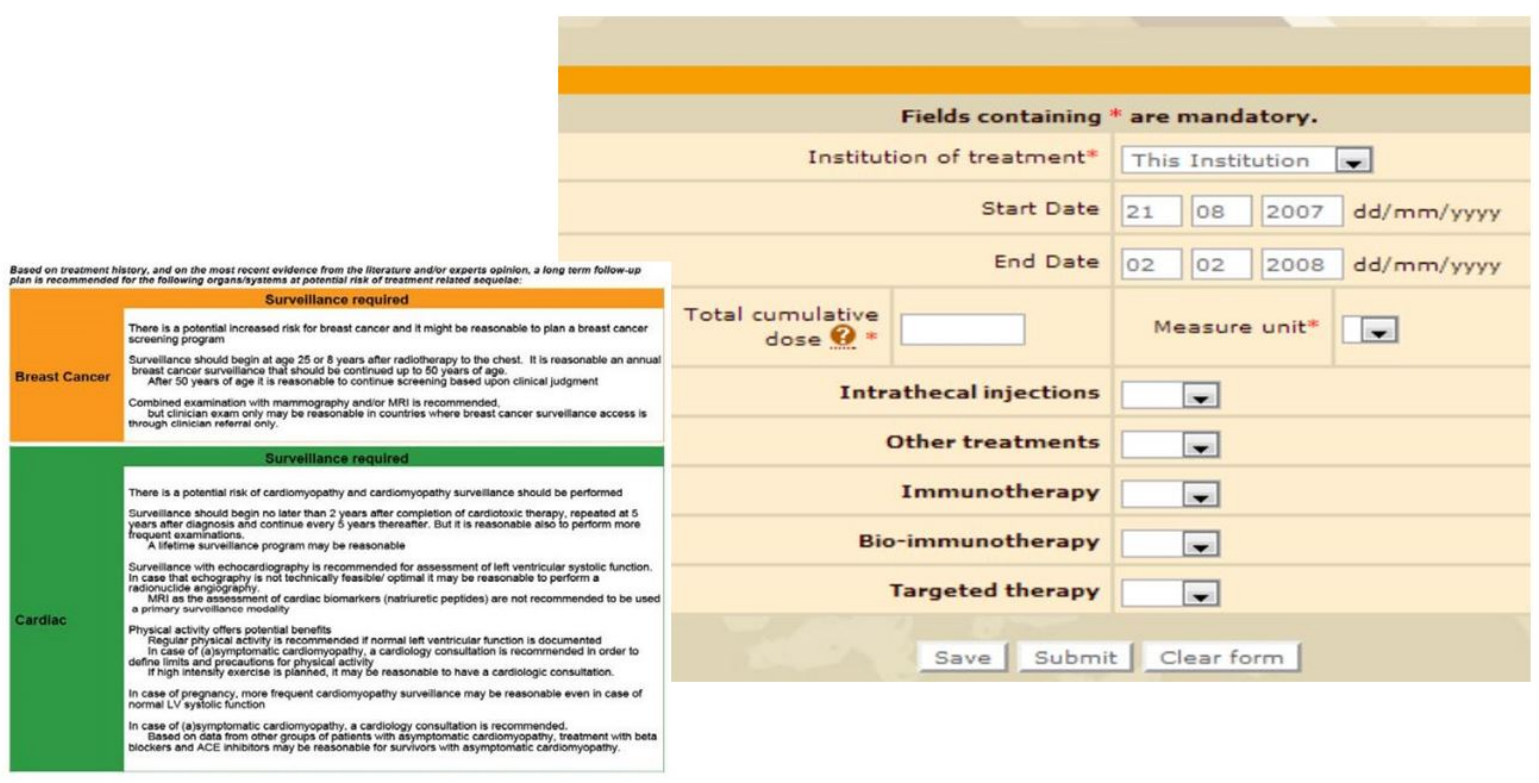

Beispiel einer Passport-Webseite mit personalisierten Informationen zu Spätfolgen

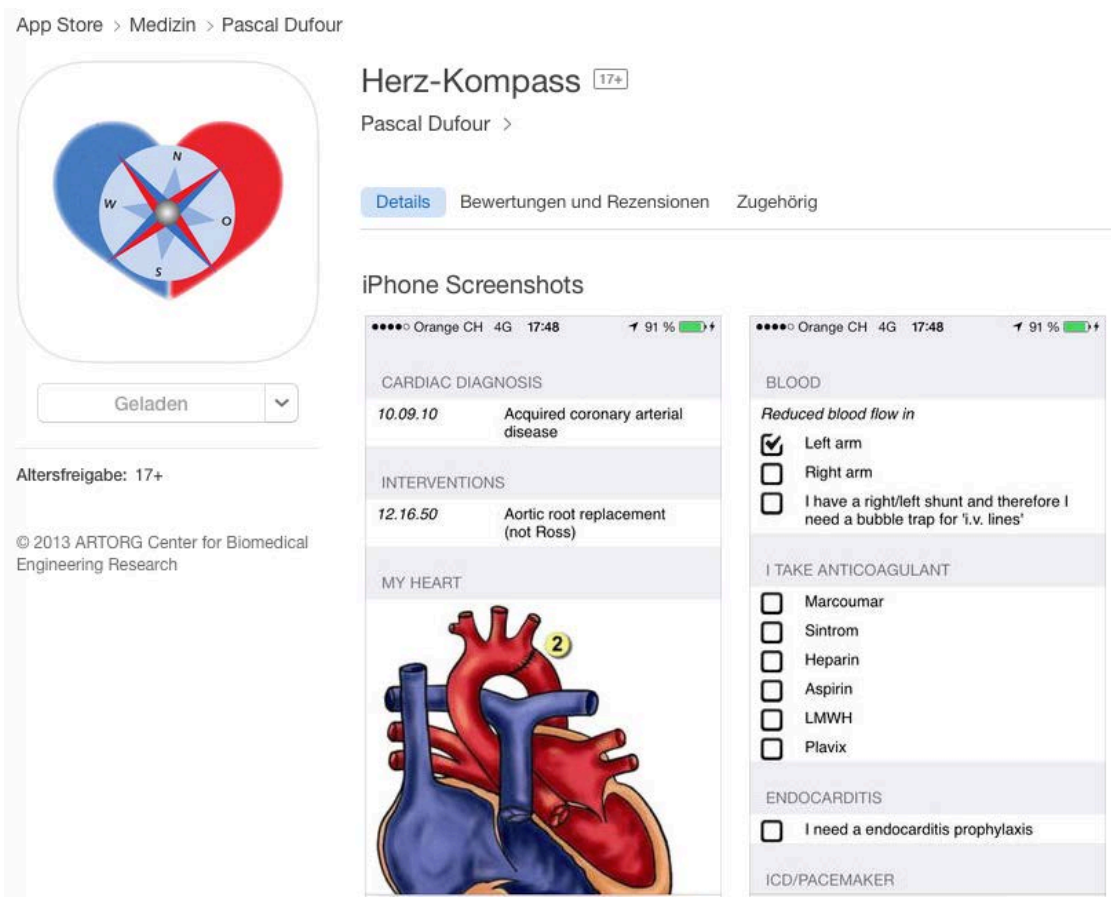

Beispiel einer medizinischen App zum Verständnis seines Herzfehlers 
Published in final edited form as:

Pädiatrie up2date 2016; 11(01): 55-74 DOI: 10.1055/s-0041-107502

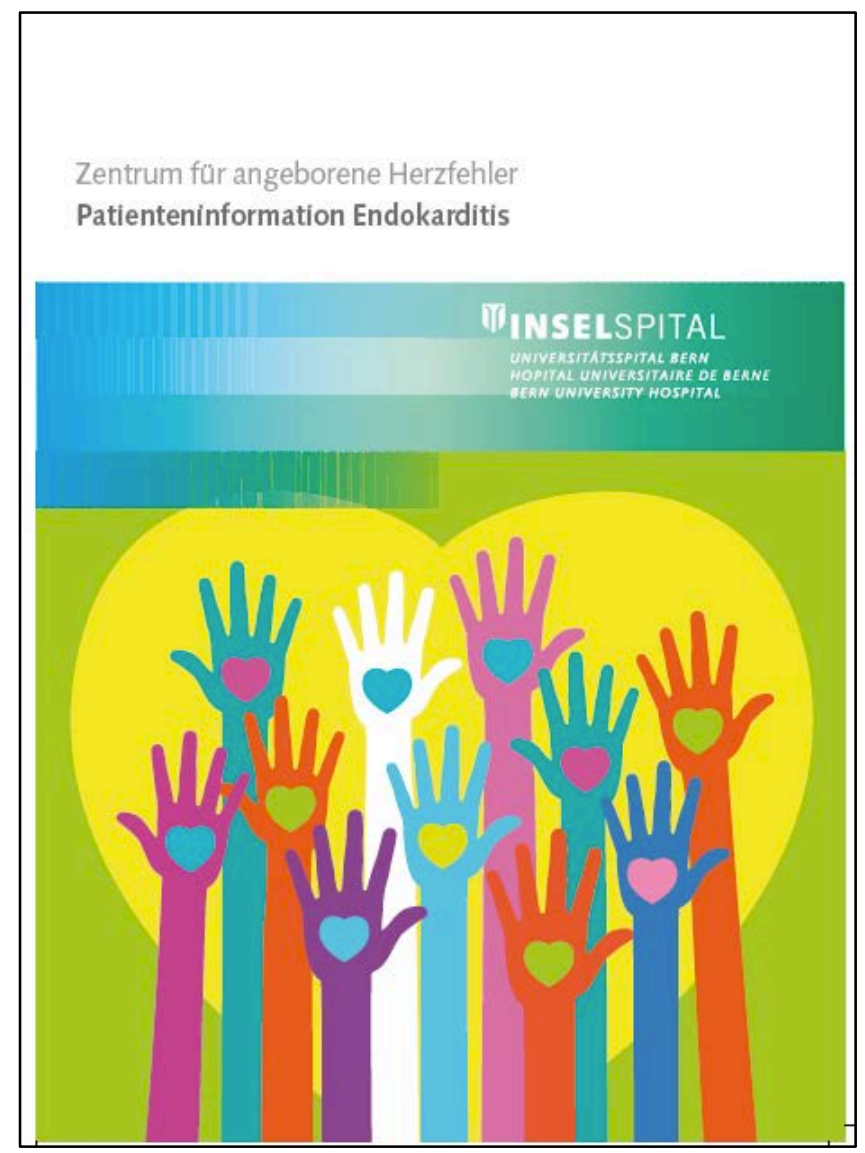

Patientenbroschüre zum Thema Endokarditis 
Published in final edited form as:

Pädiatrie up2date 2016; 11(01): 55-74 DOI: 10.1055/s-0041-107502

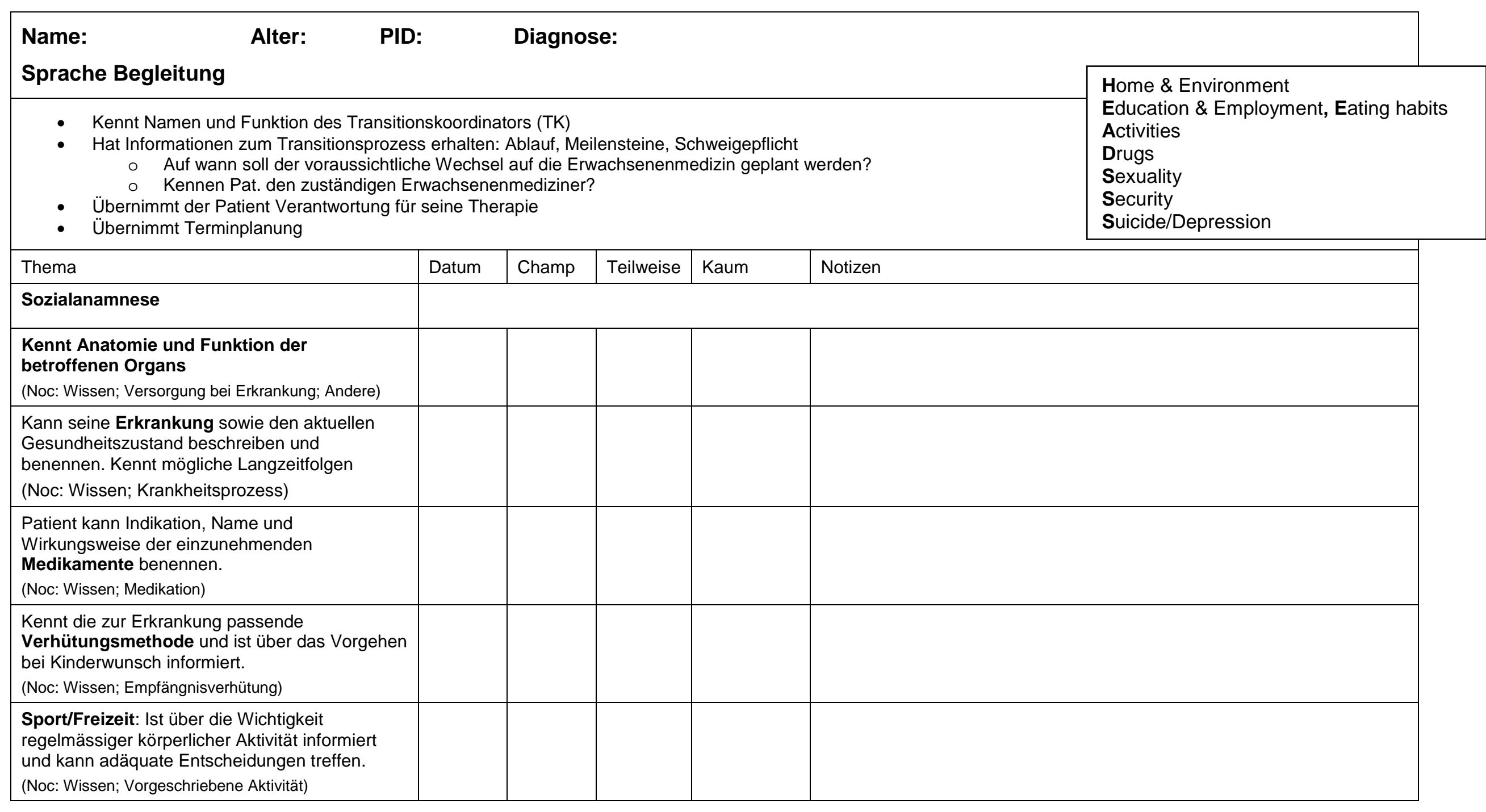


Published in final edited form as:

Pädiatrie up2date 2016; 11(01): 55-74 DOI: 10.1055/s-0041-107502

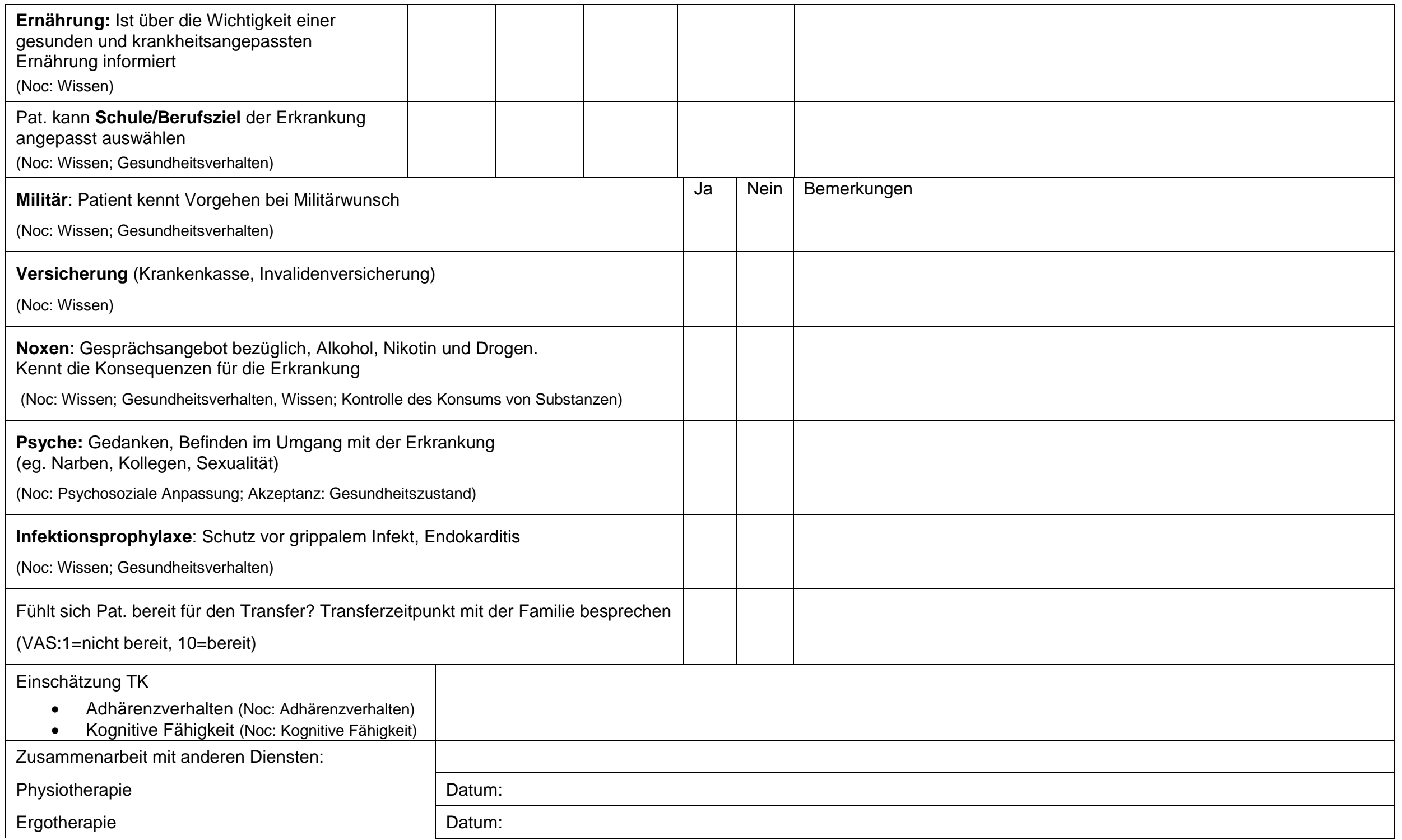


Published in final edited form as:

Pädiatrie up2date 2016; 11(01): 55-74 DOI: 10.1055/s-0041-107502

Sozialberatung

Psych. Dienste

Datum:

Datum:

Checkliste Transitions-Sprechstunde für angeborene Herzfehler, Inselspital Bern 
Published in final edited form as:

Pädiatrie up2date 2016; 11(01): 55-74 DOI: 10.1055/s-0041-107502

\section{Referenzen}

1. Michaud, P.-A., J.-C. Suris, and R. Viner, The adolescent with a chronic condition: epidemiology, developmental issues and health care provision. WHO Discussion Papers on Adolescence, World Health Organization, Geneva, 2007.

2. Michel, G., et al., Incidence of childhood cancer in Switzerland: the Swiss childhood cancer registry. Pediatric blood \& cancer, 2008. 50(1): p. 46-51.

3. Diller, L., Adult Primary Care after Childhood Acute Lymphoblastic Leukemia. New England Journal of Medicine, 2011. 365(15): p. 1417-1424.

4. Wallace, W.H.B., L. Thompson, and R.A. Anderson, Long term follow-up of survivors of childhood cancer: summary of updated SIGN guidance. Vol. 346. 2013.

5. Essig, S., et al., Risk of late effects of treatment in children newly diagnosed with standard-risk acute lymphoblastic leukaemia: a report from the Childhood Cancer Survivor Study cohort. The lancet oncology, 2014. 15(8): p. 841-851.

6. Webb, G., et al., The care of adults with congenital heart disease across the globe: Current assessment and future perspective: A position statement from the International Society for Adult Congenital Heart Disease (ISACHD). Int J Cardiol, 2015. 195: p. 326-333.

7. Menting, M.E., et al., The unnatural history of the ventricular septal defect: outcome up to 40 years after surgical closure. J Am Coll Cardiol, 2015. 65(18): p. 1941-51.

8. Schwerzmann, M., Congenital heart disease clinics - how to keep patients on board. Progress in Pediatric Cardiology, 2012. 34: p. 113-117.

9. Goossens, E., et al., Transfer of adolescents with congenital heart disease from pediatric cardiology to adult health care: an analysis of transfer destinations. J Am Coll Cardiol, 2011. 57(23): p. 2368-74.

10. Meleis, A.I., et al., Experiencing transitions: an emerging middle-range theory. Advances in nursing science, 2000. 23(1): p. 12-28.

11. Rutishauser, C. and F. Navratil, Zwischen Pädiatrie und Erwachsenenmedizinärztliche Betreuung Jugendlicher in der Schweiz. Schweiz Med Forum, 2004. 4: p. 1159-1165.

12. Paul, M., et al., Transfers and transitions between child and adult mental health services. Br J Psychiatry Suppl, 2013. 54: p. s36-40.

13. Sable, C., et al., Best practices in managing transition to adulthood for adolescents with congenital heart disease: the transition process and medical and psychosocial issues: a scientific statement from the American Heart Association. Circulation, 2011. 123(13): p. 1454-85.

14. Rutishauser, C., S.M. Sawyer, and A.E. Ambresin, Transition of young people with chronic conditions: a cross-sectional study of patient perceptions before and after transfer from pediatric to adult health care. Eur J Pediatr, 2014. 173(8): p. 1067-74.

15. Caflisch, M., Wenn chronisch kranke Jugendliche erwachsen werden. Pädiatrie, 2013. 2.

16. Tuchman, L.K., et al., Cystic Fibrosis and Transition to Adult Medical Care. Pediatrics, 2010. 125(3): p. 566-573.

17. Moceri, P., et al., From adolescents to adults with congenital heart disease: the role of transition. Eur J Pediatr, 2015. 174(7): p. 847-54.

18. Suris, J.C. and C. Akre, Key elements for, and indicators of, a successful transition: an international delphi study. J Adolesc Health, 2015. 56(6): p. 612-8.

19. Lyons, S.K., I.M. Libman, and M.A. Sperling, Diabetes in the Adolescent: Transitional Issues. The Journal of Clinical Endocrinology \& Metabolism, 2013. 98(12): p. 46394645.

20. Lugasi, T., M. Achille, and M. Stevenson, Patients' perspective on factors that facilitate transition from child-centered to adult-centered health care: a theory integrated metasummary of quantitative and qualitative studies. J Adolesc Health, 2011. 48(5): p. 429-40. 
Published in final edited form as:

Pädiatrie up2date 2016; 11(01): 55-74 DOI: 10.1055/s-0041-107502

21. American Academy of, P., et al., Supporting the health care transition from adolescence to adulthood in the medical home. Pediatrics, 2011. 128(1): p. 182-200.

22. Nagra, A., et al., Implementing transition: Ready Steady Go. Arch Dis Child Educ Pract Ed, 2015.

23. Betz, C.L. and G. Redcay, Dimensions of the transition service coordinator role. J Spec Pediatr Nurs, 2005. 10(2): p. 49-59.

24. Annunziato, R.A., et al., Strangers headed to a strange land? A pilot study of using a transition coordinator to improve transfer from pediatric to adult services. J Pediatr, 2013. 163(6): p. 1628-33.

25. Fredericks, E.M., Nonadherence and the transition to adulthood. Liver Transpl, 2009. 15 Suppl 2: p. S63-9.

26. Kovacs, A.H. and B.W. McCrindle, So hard to say goodbye: transition from paediatric to adult cardiology care. Nat Rev Cardiol, 2014. 11(1): p. 51-62.

27. American Academy of Pediatrics, American Academy of Family Physicians, and Amercian College of Physicians-American Society of Internal Medicine, A consensus statement on health care transitions for young adults with special health care needs. Pediatrics, 2002. 110(6 Pt 2): p. 1304-6.

28. Blum, R.W., Introduction. Improving transition for adolescents with special health care needs from pediatric to adult-centered health care. Pediatrics, 2002. 110(6 Pt 2): p. 1301-3.

29. Lotstein, D.S., et al., Planning for health care transitions: results from the 2005-2006 National Survey of Children With Special Health Care Needs. Pediatrics, 2009. 123(1): p. e145-52.

30. Baumann, T., Jugendliche in der «Walk-in Praxis». Praxis, 2013. 102(18): p. 11291135. 